Max-Planck-Institut für demografische Forschung

Max Planck Institute for Demographic Research

Konrad-Zuse-Strasse $1 \cdot$ D-18057 Rostock - GERMANY

Tel +49 (0) 3812081 - 0; Fax +49 (0) 3812081 - 202;

http://www.demogr.mpg.de

MPIDR WORKING PAPER WP 2004-029

DECEMBER 2004

\title{
Mortality in varying Environment
}

M. S. Finkelstein (finkelstein@ demogr.mpg.de)

This working paper has been approved for release by: James W. Vaupel (jwv@ demogr.mpg.de)

Head of the Laboratory of Survival and Longevity.

(C) Copyright is held by the authors.

Working papers of the Max Planck Institute for Demographic Research receive only limited review. Views or opinions expressed in working papers are attributable to the authors and do not necessarily reflect those of the Institute. 


\title{
Mortality in varying Environment
}

\author{
M.S. Finkelstein \\ Department of Mathematical Statistics \\ University of the Free State \\ PO Box 339, 9300 Bloemfontein, \\ Republic of South Africa \\ and The Max Planck Institute for Demographic Research \\ Konrad-Zuse-Strasse 1 \\ 18057 Rostock, Germany \\ FinkelM.SCI@mail.uovs.ac.za
}

Summary. An impact of environment on mortality, similar to survival analysis, is often modelled by the proportional hazards model, which assumes the corresponding comparison with a baseline environment. This model describes the memory-less property, when the mortality rate at a given instant of time depends only on the environment at this instant of time and does not depend on the history. In the presence of degradation the assumption of this kind is usually unrealistic and historydependent models should be considered. The simplest stochastic degradation model is the accelerated life model. We discuss these models for the cohort setting and apply the developed approach to the period setting for the case when environment (stress) is modelled by the functions with switching points (jumps in the level of the stress).

\section{Introdiction}

The process of human aging is a process of accumulation of damage of some kind (e.g., accumulation of deleterious mutations). It is natural to model it via some stochastic process. Death of an organism uniquely defines the corresponding lifetime random variable in a cohort setting. We are interested in an impact of varying environment on the mortality rate, which is defined for a cohort via the lifetime distribution function in a standard way. There are two major possibilities. The first one is plasticity: a memory-less property, which says that mortality rate does not depend on the past trajectory of an environment and depends only on its current value. This is the unique property in some sense and a widely used proportional hazards $(\mathrm{PH})$ model is a conventional tool for modelling plasticity. On the other hand, dependence on history is more natural for the hazard (mortality) rate of degrading objects, as it seems reasonable that the chance to fail in some small interval of time 
is higher for objects with higher level of accumulated degradation. There are various ways of modelling this dependence. The simplest one is via the accelerated life model (ALM), which performs the scale transformation in the lifetime distribution function. The ALM can be equivalently defined via the mortality rates as well (see Section 1).

These two models and their generalizations were thoroughly investigated in reliability and survival analysis studies (Bogdanovicius and Nikulin, 2002), where the cohort setting is a natural one for defining the corresponding lifetime random variables. In Section 2 we discuss some traditional and new results for a cohort setting. In demography, however, period mortality rates play a crucial role, whereas defining 'proper' lifetime random variables is not straightforward and needs additional assumptions on a population structure. We mostly focus on the case when environment has switching points: jumps in severity from one level to another but the situation without switching points is also discussed. Generalization of the $\mathrm{PH}$ model to the period case is quite natural, whereas the corresponding generalization of the ALM needs careful reasoning. In Section 3 we perform this operation explicitly for the case of the linear ALM and discuss the idea how it can be generalized to the time-dependent scale transformation.

\section{Damage accumulation and plasticity. Cohort setting}

\subsection{Proportional hazards}

Denote by $X$ a cohort lifetime random variable (age at death) and by $\mu(x)$ and $l(x)$ the corresponding mortality rate and the survival probability, respectively. Then:

$$
\bar{F}(x) \equiv l(x)=\exp \left\{-\int_{0}^{x} \mu(u) d u\right\},
$$

where $F(x)$ is the cumulative lifetime distribution function (Cdf) and $\bar{F}(x)=$ $1-F(x)$.

Let $z(x), x \geq 0$ be an explanatory variable, which for simplicity is assumed to be a scalar one. The function $z(x)$ describes environment or stress. We want to model an impact of a stress (environment) on $X$. Consider two stress functions: $z_{0}(x)$ and $z(x)$ - the baseline and the current, respectively. The stress $z_{0}(x)$ is an arbitrary function from the family of all admissible stresses $A$. The stress $z_{0}(x) \in A$ is usually a fixed function. Denote the mortality rate and the Cdf under the baseline stress by $\mu_{0}(x)$ and $F_{0}(x)$, respectively, and under the current stress, as in equation (1), by $\mu(x)$ and $F(x)$, respectively.

The most popular way to model a stress impact is via the PH model:

$$
\mu(x)=w_{P}(z(x)) \mu_{0}(x)
$$

where $w_{P}(z(x))$ is a positive, strictly monotone, function (usually unknown), the sub-script " $P$ " stands for "proportional" and $w_{P}\left(z_{0}(x)\right) \equiv 1$. 
Consider now a step stress with switching from the baseline to the current stress at some $x_{s}>0$. Several switching points can be considered similarly. This step stress models the abrupt change in environment (e.g., the development of a new critical for the healthcare drug, or the dramatic change in the lifestyle):

$$
z_{s}(x)= \begin{cases}z_{0}(x), & 0 \leq x<x_{s} \\ z(x), & x_{s} \leq x<\infty\end{cases}
$$

In accordance with definition (2), the mortality rate $\mu_{s}(t)$ for the stress $z_{s}(x)$ is:

$$
\mu_{s}(x)= \begin{cases}\mu_{0}(x), & 0 \leq x<x_{s} \\ \mu(x), & x_{s} \leq x<\infty\end{cases}
$$

Therefore, the change point in a stress results in the corresponding change point in $\mu_{s}(t)$ : instantaneous jump to the level $\mu(x)$.

Definition (2) and properties (3)-(4) show that a plastic, memory-less reaction of the mortality rate on the changes in the stress function takes place. Denote by $F_{s}(x)$ the Cdf, which corresponds to the mortality rate $\mu_{s}(t)$. The remaining lifetime also does not depend on the mortality rate history in $\left[0, x_{s}\right)$, as clearly follows from the equation for the remaining lifetime $\operatorname{Cdf} F_{r s}\left(x \mid x_{s}\right)$ :

$$
\bar{F}_{r s}\left(x \mid x_{s}\right) \equiv \frac{\bar{F}_{s}\left(x+x_{s}\right)}{\bar{F}_{s}\left(x_{s}\right)}=\exp \left\{-\int_{x_{s}}^{x+x_{s}} \mu(u) d u\right\} .
$$

The PH model is usually not suitable for modelling an impact of stress on degrading (aging) objects, as it means that the stress in $[0, x)$ does not influence the degradation process in $(x, \infty)$. This assumption usually does not hold as the past changes in stress affect the history of the degradation process, changing its current value. These considerations, of course, are valid for any memory-less model (see the next section).

Mortality rates of humans are increasing in age $x$ (for adults) as the consequence of biological degradation processes. However, there is at least one but a very important for the topic of our paper case which shows that the $\mathrm{PH}$ model can be used for the human cohort mortality rate modelling as well. In this case the notion of stress has a more general meaning.

Example 1. Lifesaving. Describe the mortality environment for a population via the quality of a healthcare. Let $\mu_{0}(x)$, as previously, denote the mortality rate for some baseline, standard level of healthcare. Suppose that the better level of health care had been achieved, which usually results in lifesaving (Vaupel and Yashin, 1987)): each life, characterized by the initial mortality rate $\mu_{0}(x)$ is saved (cured) at each event of death with probability $1-\theta(x), 0<\theta(x) \leq 1$ (or, equivalently, this proportion of individuals who would have died are now resuscitated and given another chance). Those who are saved, experience the minimal repair. The minimal repair is defined (Finkelstein, 2000), as the repair that brings an object back to the state it 
had just prior to the failure (death). It is clear that the new healthcare environment defined in such a way does not change the process of individual aging. If $\theta(x)=0$, the lifetime is infinite and virtual deaths' form a memoryless nonhomogenous Poisson process. It can be proved (Vaupel and Yashin, 1987; Finkelstein, 1999) that under given assumptions the new mortality rate is given by:

$$
\mu(x)=\theta(x) \mu_{0}(x),
$$

which is the specific form of the PH model (2). The case, when there is no cure $(\theta(x)=1)$, corresponds to the baseline mortality rate $\mu_{0}(x)$ and switching from the "stress" $\theta(x)=1$ to the stress $0<\theta(x)<1$ at age $x_{s}$ results in the plasticity property given by equation (4).

Note, that the baseline mortality rate $\mu_{0}(x)$ can also model a possibility of lifesaving. In this case $\mu(x)$ defines the larger probability of lifesaving. Formally, the hypothetical mortality rate without lifesaving $\mu_{h}(x)$ should be then defined:

$$
\mu_{0}(x)=\theta_{h}(x) \mu_{h}(x), \quad 1-\theta_{h}(x)<1-\theta(x), x>0 .
$$

The switching point in lifesaving, in fact, means that at a certain age $x_{s}$ a switch from one probability of lifesaving to another is performed.

\subsection{Accelerated life model}

Another popular model describing an impact of a stress on $X$ is the accelerated life model (ALM) (Cox and Oakes, 1984; Finkelstein, 1999). It performs the stress-dependent scale transformation of the baseline Cdf

$$
F_{0}(x)=1-\exp \left\{-\int_{0}^{x} \mu_{0}(u) d u\right\}
$$

in the following way:

$$
F(x)=F_{0}\left(\int_{0}^{x} w_{A}(z(u)) d u\right) \equiv F_{0}\left(W_{A}(x)\right),
$$

where the subscript "A" stands for "accelerated", and notation

$$
\int_{0}^{x} w_{A}(z(u)) d u=W_{A}(x)
$$

is used for convenience. As previously, we assume that $w_{A}\left(z_{0}(x)\right) \equiv 1$. Note that $w_{A}(z(x))$ is unknown but can be estimated from the data.

This model is usually more appropriate for modelling additive degradation (accumulation of damage), as the effect of higher stress with $w(z(x))>1$, for instance, results in facilitation of degradation processes. The function $w(z(x))$ can be interpreted as a rate of degradation, whereas $W_{A}(x)$ is the accumulated 
damage in this case. We shall also assume in this model that mortality rates are increasing, as monotone degradation usually can be described by IFR (increasing failure rate) lifetime distributions. The mortality rate is obtained from equation (7) as (compare with equation (2)):

$$
\mu(x)=w_{A}(z(x)) \mu_{0}\left(W_{A}(x)\right) .
$$

Similar to equation (5) the survival function for the remaining lifetime is:

$$
\begin{aligned}
\bar{F}_{r}(x \mid a) & \equiv \frac{\bar{F}(x+a)}{\bar{F}(a)}=\frac{\bar{F}_{0}\left(W_{A}(x+a)\right)}{\bar{F}_{0}\left(W_{A}(a)\right)} \\
& =\frac{\bar{F}_{0}\left(W_{A}(a)+\int_{0}^{x} w_{A}(z(u+a)) d u\right)}{\bar{F}_{0}\left(W_{A}(a)\right)}, \quad a>0,
\end{aligned}
$$

where an important for the model additivity property is used:

$$
\int_{0}^{x+a} w_{A}\left(z(u) d u=W_{A}(a)+\int_{a}^{x+a} w_{A}(z(u)) d u\right.
$$

Unlike equation (5)), the remaining lifetime already depends on the mortality rate history in $[0, a)$, but this dependence is only on the simple aggregated history characteristic $W_{A}(x)$.

Let the true' biological age $x$ be defined for the baseline stress $z_{0}(x)$, then the virtual age in the baseline environment of an organism that had survived time $x$ under the current stress $z(x)$, in accordance with ALM, is defined as (Finkelstein, 1992, Kijima, 1988):

$$
x_{V}=W_{A}(x),
$$

and the corresponding difference between these two ages is:

$$
\triangle_{V} \equiv x_{V}-x .
$$

Therefore, the ALM gives a simple and effective way for age correspondence under different stresses. If an organism had survived time $x$ under the baseline stress, his virtual age under the current stress is $W_{A}^{-1}(x)$. Note that for the $\mathrm{PH}$ model the virtual age is equal to the calendar one.

If $w_{A}(z(x))>x, \forall x>0$, then $W_{A}(x)>x$ and the stress $z(x)$ is more severe than the baseline one, which in accordance with equation (10) means that $x_{V}>x$. Additionally, the corresponding mortality rates are ordered in this case as:

$$
\mu_{0}(x)<\mu(x), \quad \forall x>0,
$$

which for increasing $\mu_{0}(x)$ immediately follows from equation (8).

Definition (7) reads:

$$
\exp \left\{-\int_{0}^{x} \mu(u) d u\right\}=\exp \left\{-\int_{0}^{W_{A}(x)} \mu_{0}(u) d u\right\}
$$


and

$$
\int_{0}^{x} \mu(u) d u=\int_{0}^{W_{A}(x)} \mu_{0}(u) d u .
$$

Therefore, given the mortality rates under two stresses in $[0, \infty)$, the function $W_{A}(x)$ can be obtained.

Similar to the previous subsection, consider now the stress $z_{s}(x)$ defined by equation (3) and assume for the definiteness that $z(x)$ is more severe than $z_{0}(x)$. The corresponding $\operatorname{Cdf} F_{s}(x)$ for this stress is:

$$
F_{s}(x)= \begin{cases}F_{0}(x), & 0 \leq x<x_{s} \\ F_{0}\left(x_{s}+\int_{x_{s}}^{x} w_{A}(z(u)) d u\right), & x_{s} \leq x<\infty .\end{cases}
$$

Transforming the second row in equation (13):

$$
\begin{aligned}
F_{0}\left(x_{s}+\int_{x_{s}}^{x} w_{A}(z(u)) d u\right) & =F_{0}\left(\int_{x_{s}-\tau}^{x} w_{A}(z(u)) d u\right) \\
& =F_{0}\left(W_{A}\left(x_{s}\right)-W_{A}\left(x_{s}-\tau\right)\right),
\end{aligned}
$$

where $\tau$ is uniquely defined from the equation:

$$
x_{s}=\int_{x_{s}-\tau}^{x_{s}} w_{A}(z(u)) d u .
$$

Thus, the virtual age under the stress $z(x)$ (in other words, the re-calculated for the more severe stress the baseline age' $x_{s}$ ) just after the switching is $x_{s}-\tau$. Equation (15) defines an interval $\left[x_{s}-\tau, x_{s}\right)$ in which the accumulated degradation under the stress $z(x)$ is equal to the accumulated degradation $x_{s}$ under the stress $z_{0}(x)$ in the interval $\left[0, x_{s}\right)$.

A jump in the stress at $x_{s}$ leads to a jump in mortality rate, which can be clearly seen by comparing equation (8) with

$$
\mu_{s}(x)= \begin{cases}\mu_{0}(x), & 0 \leq x<x_{s} \\ w_{A}(z(x)) \mu_{0}\left(x_{s}+\int_{x_{s}}^{x} w_{A}(z(u)) d u\right), & x_{s} \leq x<\infty\end{cases}
$$

as for increasing $\mu_{0}(x)$ and for $w_{A}(z(x))>1, x \in\left[x_{s}, \infty\right)$ :

$$
\begin{aligned}
\mu_{0}(x) & <w_{A}(z(x)) \mu_{0}\left(x_{s}+\int_{x_{s}}^{x} w_{A}(z(u)) d u\right) \\
& <w_{A}(z(x)) \mu_{0}\left(\int_{0}^{x} w_{A}(z(u)) d u\right) \\
& =w_{A}(z(x)) \mu_{0}\left(W_{A}(x)\right)=\mu(x) .
\end{aligned}
$$

Inequality (16) is a special case of inequality (11), obtained for a more severe stress $z_{s}(x)$. 
It is important to note that, as follows from relations (7) and (14), for the general case $F_{0}\left(x_{s}+\int_{x_{s}}^{x} w_{A}(z(u)) d u\right)$ is not a segment of $F(x)$ for $x \geq x_{s}$ (and the corresponding mortality rate is not a segment of $\mu(x)$ ), but for the specific linear case $W_{A}(x)=w_{A} t$ it can be transformed to a segment:

$$
F_{0}\left(w_{A} \cdot\left(x-x_{s}+\tau\right)\right)=F\left(x-x_{s}+\tau\right),
$$

where $\tau$ is obtained from a simplified equation:

$$
x_{s}=\int_{x_{s}-\tau}^{x_{s}} w_{A} d u=\int_{0}^{\tau} w_{A} d u \Rightarrow \tau=\frac{x_{s}}{w_{A}}, w_{A}>1
$$

and, finally, only for this specific linear case the Cdf (13) can be defined in the way usually referred to in the literature (Nelson, 1993):

$$
F_{s}(x)= \begin{cases}F_{0}(x), & 0 \leq x<x_{s} \\ F\left(x-x_{s}+\tau\right), & x_{s} \leq x<\infty\end{cases}
$$

Sometimes this equation written in terms of mortality rates:

$$
\mu_{s}(x)= \begin{cases}\mu_{0}(x), & 0 \leq x<x_{s} \\ \mu\left(x-x_{s}+\tau\right), & x_{s} \leq x<\infty\end{cases}
$$

is called the 'Sedjakin principle', although Sedjakin (1966) defined it in a more general way as the dependence on history only via the accumulated mortality rate. As $w_{A}=$ const, $\mu(x)$ is also an increasing function. Taking into account that $\tau<x_{s}$ :

$$
\mu_{s}(x)=\mu\left(x-x_{s}+\tau\right)<\mu(x), \quad x_{s} \leq x<\infty,
$$

which is a specific case of inequality (16).

\subsection{Other models}

There are not so many other candidates for memory-less models, the additive hazard $(\mathrm{AH})$ model being probably the only one, which is widely used in applied statistical analysis:

$$
\mu(x)=\mu_{0}(x)+w_{A D}(z(x)),
$$

where $w_{A D}(x)$ is a positive function $\left(w_{A D}\left(z_{0}(x)\right) \equiv 1\right)$ and the subscript "AD" stands for "additive". It is clear that the plasticity property (4), defined for the stress given by equation (3), holds also for this case. Similar to the PH model the stress in $[0, x)$ does not influence the degradation process in $(x, \infty)$, but, probably, the AH model is more suitable when, for instance, the baseline 
$\mu_{0}(x)$ describes some 'inherent' degradation process which is not influenced by the environment.

The memory-less property is a rather unique feature, whereas the dependence on a history can be modelled in numerous ways. Most of these generalizations are based on different extensions of the ALM or of the PH model (Bogdanovicius and Nikulin, 2002). For instance, equation (8) can be generalized to:

$$
\mu 8 x)=G\left(z(x), w_{A}\left(z(x), W_{A}(x)\right)\right)
$$

where $G(\cdot)$ is a positive function. The advanced statistical methods of analyzing the data via the chosen model also can be found in (Bogdanovicius and Nikulin, 2002). Our goal in this paper is, however, to discuss plasticity versus accumulated damage modelling for mortality rates in the cohort and period settings. The ALM is just a tractable example, which can be used for degradation modelling.

Let, as previously, $\mu_{0}(x)$ and $\mu(x)$ be two mortality rates for populations at baseline and current stresses, respectively. Assume that the rates are given or observed and this is the only information at hand. It is clear that without additional information on the degradation process or on the possible memorylees property the 'proper' model for the stress influence is non-identifiable, as different models can result in the same. Indeed, by letting $w_{P}(z(x))=$ $\mu(x) / \mu_{0}(x)$ we arrive at the $\mathrm{PH}$ model (2), and by obtaining $W_{A}(x)$ from equation (12), which is always possible, results in the ALM (7). The following simple illustrative example will be also helpful for the reasoning of the next section.

\section{Example 2. The Gompertz curve}

Let

$$
\begin{gathered}
\mu_{0}(x)=a \exp \{b x\}, \quad a, b>0 \\
\mu(x)=w_{P} \mu_{0}(x), \quad w_{P}>0
\end{gathered}
$$

Therefore, equations (21) and (22) formally describe the PH model with a constant in age factor $w_{P}$. On the other hand, assuming the ALM defined by equation (7), the function $W_{A}(x)$ can be obtained from equation (12):

$$
\int_{0}^{x} \mu(u) d u=\int_{0}^{W_{A}(x)} \mu_{0}(x) d u \Rightarrow w_{P}(\exp \{b x\}-1)=\exp \left\{b W_{A}(x)\right\}-1 .
$$

In accordance with the contemporary mortality data for the developed countries (Boongaarts and Feeney, 2002) parameter $b$ is approximately estimated as 0.1. Equation (22) can be simply approximately solved with a sufficient accuracy for $x>30$ (when aging starts and the Gompertz curve is suitable for modelling):

$$
W_{A}(x) \approx \frac{\ln w_{P}}{b}+x .
$$


If $w_{P}<1$, a condition: $x>30$ in combination with real values of parameters guarantees that $W_{A}(x)>0$. Therefore, the ALM defined by relation (23) can formally explain equations (21) and (22), although it is not clear how to explain that the difference between the virtual and baseline ages $\triangle_{V}$, defined by equation (11), is approximately constant for this model. An explanation via the $\mathrm{PH}$ model seems much more natural.

If there is no sufficient information on the 'physical' processes of degradation in our objects, the simplest way to distinguish between the memory-less and accumulation of degradation models is to conduct an experiment and to apply the stress $z_{s}(x)$, defined by equation (3), to our cohort. If the resulting mortality rate $\mu_{s}(x)$ is obtained in the form, defined by equation (4), then we arrive at a memory-less property, which means that our object is 'degradation free'. The other option is that there is no dependence on the history of this degradation like in the lifesaving model or the degradation described by the baseline $\mu_{0}(x)$ does not depend on the environment. The latter possibility was already mentioned while discussing the AH model. On the other hand, if there is a dependence on the degradation history, then the resulting mortality rate should be

$$
\mu_{s}(x)= \begin{cases}\mu_{0}\left(x_{s}\right), & 0 \leq x<x_{s} \\ \tilde{\mu}\left(x_{s}\right), & x_{s} \leq x<\infty\end{cases}
$$

where the mortality rate $\tilde{\mu}(x)$, e.g., for the ALM, as follows from inequality (16), is contained between baseline and 'current' mortality rates:

$$
\mu_{0}(x)<\tilde{\mu}(x)<\mu(x), \quad x_{s} \leq x<\infty .
$$

For a general case, if accumulated degradation in $\left[0, x_{s}\right)$ under the stress $z_{0}(x)$ is smaller than under the stress $z(x)$, inequality (25) should be considered as a reasonable assumption.

Inequality (16) defines a jump in mortality rate, which corresponds to a jump in the stress. For a general case the reaction in mortality rate should not be necessarily in the form of the jump: it can be some smooth function, showing some 'inertia' in the degradation process.

In simple electronic devices without degradation the failure rate pattern usually follows the stress pattern. In the lifesaving PH model, however, it is not often the case, as environmental changes are usually rather smooth which results in the smooth change in the probability of lifesaving. An important feature is that after some delay the mortality rate $\mu_{s}(x), x>x_{s}$ reaches the level of $\mu(x)$. (Alternatively this delay can be modelled in the degradation framework with a short-term memory of the history of the degradation process).

The relevant example is the convergence of mortality rates of 'old cohorts' after unification of east and West Germany at $x_{s}=1990$. ((Vaupel et al, 2003). This, of course is the consequence of a direct (better healthcare) and of an indirect (better environment eliminates some causes of death) lifesaving. 
Another memory-less example, which is more likely to be modelled by the AH model, is the dietary restriction in Drosophila (Mair et al). The results of this paper show practically absolute plasticity: the age-specific mortality of the flies with dietary restriction depends only on their age and their current nutritional status, with past nutrition having no detectable effect.

\subsection{Damage accumulation and plasticity. Period Setting}

The detailed modelling of the previous section is essential for considering the PH model and the ALM for the period setting. As far as we know, this topic was not considered in the literature. Denote by $N(x, t)$ a population density (age-specific population size) at time $t$ - a number of persons of age $x$. See Keding (1990) and Arthur and Vaupel (1984) for discussion of this quantity. We shall call $N(x, t), x \geq 0$ a population age structure at time $t$. Let $\mu(x, t)$ denote the mortality rate as a function of age $x$ and time $t$ for a population with the age structure $N(x, t), x \geq 0$ :

$$
\mu(x, t)=\lim _{\delta \rightarrow 0} \frac{(N(x+\delta, t+\delta)-N(x, t)) / d \delta}{N(x, t)}
$$

On the other hand, it is clear that, as $\mu(x, t) d \delta$ is a local risk of death, it, in fact, does not depend on $N(x, t), x \geq 0$. This means that for defining the $\mathrm{PH}$ model we do not need to define the corresponding lifetime variable. The stress now is a function of time: $z(t)$, and the cohort PH model (2) is generalized to:

$$
\mu(x, t)=q_{P}(z(t), x) \mu_{0}(x, t) .
$$

If the stress (environment) is constant, the mortality rate does not depend on time and the population is stationary with additional assumptions that it is closed to migration and experience a constant birth rate. Consider now a step function in time $t$, which is a special case of the stress (3):

$$
\tilde{z}(t)= \begin{cases}z_{0}, & 0 \leq t<t_{s} \\ z, & t_{s} \leq t<\infty\end{cases}
$$

Assuming the constant in age $x \mathrm{PH}$ model, the mortality rate for this stress is given by (compare with equations (2), (3) and (4)):

$$
\mu_{s}(x, t)=\left\{\begin{array}{ll}
\mu_{0}(x), & 0 \leq t<t_{s} ; x \geq 0 \\
w_{P}(z) \mu_{0}(x), & t_{s} \leq t<\infty ; x \geq 0
\end{array} .\right.
$$

Therefore, the baseline mortality rate after the change point is multiplied by $w_{P}(z)$ for all ages and not for the interval of ages as in equation (4). This is an important distinction from the step stress modelling in the cohort setting. The other important 'negative' feature of the period setting is that now the experiment with a step stress without analyzing concrete cohorts (see later) 
cannot indicate the memory-less property (if any) in a way it did for the purely cohort setting. (Note, that similar to (28), the AH model results for $t \geq t_{s}$ in the mortality rate $\left.\mu_{0}(x)+w_{A D}(z)\right)$.

The period ALM at time $t$ should be applied to each cohort with varying age $x(0 \leq x<\infty)$ at time $t$, and we must assume, as previously, that our population is closed to migration and experience a constant birth rate. In this case the corresponding lifetime random variable for each cohort is properly defined and the population is stationary before the change point and after it as well. We shall illustrate the construction of the period ALM for the step stress (3), where, as previously, the stress $z$ is a more severe than the stress $z_{0}$. The opposite ordering of stresses is considered in the same way. Due to piecewise constant stress, the linear ALM with a constant rate $w_{A}$ can be used and the mortality rate is defined via equation similar to equation (18) but with the age and time-dependent shift $\tau(x, t)$ :

$$
\mu_{s}(x, t)= \begin{cases}\mu_{0}(x), & 0 \leq t<t_{s} ; \quad x \geq 0 \\ \mu(x+\tau(x, t)), & t_{s} \leq t<\infty ; \quad x \geq 0\end{cases}
$$

where $\tau(x, t)$ is obtained from equation similar to equation (17):

$$
x-I\left(t-t_{s}\right)=\int_{x-\tau(x, t)}^{x} w_{A} d u, \quad w_{A}>1,
$$

where $I\left(t-t_{s}\right)$ is an indicator:

$$
I\left(t-t_{s}\right)=\left\{\begin{array}{ll}
0, & 0 \leq t<t_{s} \\
1, & t_{s} \leq t<\infty
\end{array} .\right.
$$

Equation (30) has the following solution:

$$
\tau(x, t)= \begin{cases}\frac{x-\left(t-t_{s}\right)+\left(t-t_{s}\right) w_{A}}{w_{A}}, & x>\left(t-t_{s}\right) \\ 0, & x \leq\left(t-t_{s}\right) .\end{cases}
$$

Specifically, when $t=t_{S}$ similar to equation (17): $\tau\left(x, t_{s}\right)=s / w_{A}$, but now this solution is valid for all ages $x$. Therefore, for each $t>t_{s}$ the recalculation of initial age $\tau\left(x, t_{s}\right)$ is performed for each cohort. Specifically, if the age of the cohort is less than $t-t_{S}$ and therefore this cohort was born after the change point $t_{S}$ and 'does not re-member' the previous stress $z_{0}$. All possibilities are incorporated by equation (29). An importance of the switching strategy is again in the fact that, if we look at concrete cohorts in the period framework, we are still able to detect the memory-less property or the absence of it.

A cumbersome generalization of this approach to the general time-dependent stress can be also performed using the similar considerations: at each time $t$ the initial age $\tau(x, t)$ is obtained using the following expression for the age structure of a closed to migration population: 


$$
N(x, t)=B(t-x) \exp \left\{-\int_{0}^{x} \mu(u, t-x+u) d u\right\} .
$$

where $B(t-x)$ is the birth rate at time $(t-x)$.

Considering the time-dependent stress for the $\mathrm{PH}$ model, however, is much simpler. For the case with a switching point equations (27) and (28) is generalized to:

$$
\mu_{s}(x, t)= \begin{cases}\mu_{0}(x, t), & 0 \leq t<t_{s} ; \quad x \geq 0 \\ w_{P}(z(t), x) \mu_{0}(x, t), & t_{s} \leq t<\infty ; \quad x \geq 0\end{cases}
$$

where the multiplier already depends on the stress at time $t$ and on the age $x$.

Different environments can be defined not necessarily by the switching point or by considering changing in time stresses. Let the stress $z_{0}\left(t_{0}\right)$ be a baseline stress at a baseline (fixed) time instant $t_{0}$. Denote the corresponding mortality rate, as previously, by $\mu_{0}\left(x, t_{0}\right)$. Then the stress $z_{0}\left(t_{0}\right)$ and the mortality rate $\mu(x, t)$ characterize the current instant of time $t$. Note that in this approach populations can be different and $t-t_{0}$ can be reasonably large (e.g., 10 or 20 years). The PH model for this case is naturally defined as (compare with (27)):

$$
\mu(x, t)=w_{P}(z(t), x) \mu_{=}\left(x, t_{0}\right), \quad x \geq 0, t>t_{0},
$$

where $\mu_{0}\left(x, t_{0}\right)$ plays the role of a baseline mortality rate. The analogue of the ALM, however, is not straightforward, as there should be a pair wise comparison between the corresponding cohorts of the same age $x$, using expression (32) for both instants of time. This topic needs further study.

Example 3. Gompertz shift model As stated in Bongaarts and Feeney (2002), the mortality rate in contemporary populations with high level of life expectancy tends to improve over time by a similar factor at all adult ages which results in our notation in the following Gompertz shift model (similar to equations (21) and (22)):

$$
\begin{aligned}
\mu_{0}\left(x, t_{0}\right) & =a \exp \{b x\}, \quad a, b>0, \\
\mu(x, t) & =w_{P}(z(t)) \mu_{0}\left(x, t_{0}\right), \quad w_{P}>0,
\end{aligned}
$$

This model was verified using contemporary data for different developed countries. Equations (35) and (36) define formally the age independent PH model. We do not have a switching in stress here which could help in verifying plasticity.

Most researchers agree that the process of human aging is the process of accumulation of damage of some kind (e.g., accumulation of deleterious mutations). Given the reasoning of the previous sections it means that the $\mathrm{PH}$ model (35), (36) is not suitable for this case unless it describes the lifesaving 
model. On the other hand, as it was stated in Example 2, it is really unnatural trying to explain (35)-(36) via some degradation model. Therefore, if the linear trend takes place (or, equivalently, the logarithms of mortality rates at different time instants are practically parallel), this can be explained by lifesaving in a general sense and not by slowing down the degradation processes, for instance. In other words: lifesaving is likely to be the main source of lifespan extension at present time (according to the data: at least, since the second half of the previous century). Of course, it is not a strict statement, but just a reasoning that seems to be true.

\section{Concluding remarks}

The most popular models that account for an impact of environment on a lifetime are the PH model and the ALM. The first one is the simplest way to describe the memory-less property, whereas the second describes the simplest dependence on a history in a form of accumulated damage. Various generalizations of these models are considered in Bogdanovicius and Nikulin (2002). In survival analysis these models were traditionally defined for the cohort setting.

The conventional demographic definition of the observed in a period (from $t$ to $t+\triangle t)$ age-specific and time(26). The generalization of the cohort $\mathrm{PH}$ model to this case is given by equations (27), (28) and (29). The corresponding generalization of the ALM is explicitly performed for a specific case of the step stress $\tilde{z}_{s}$. Therefore, the cohort ALM is applied to each cohort with varying age $x(0 \leq x<\infty)$ at time $t$, which results in equations (29)-(31) defining the age specific mortality rate.

Although human aging is definitely a process of damage accumulation, the contemporary demographic data supports the Gompertz shift model (35)-(36), which is, at least formally, the PH model. In line with our reasoning of the previous section this means that lifesaving (versus the decrease in the rate of degradation) can explain the decrease in mortality rates with time.

\section{References}

1. Arthur, W.B., and Vaupel, J.W. (1984). Some general relationships in population dy-namics.Population Index, 50, 214-226.

2. Bogdonavicius, V., and Nikulin, M. (2002).Accelerated Life Models. Modeling and Statistical Analysis, Chapman and Hall.

3. Bongaarts, J., and Feeney G. (2002). How long do we live? Population and Development Review 28, 13-29.

4. Cox, D.R., and Oakes D. (1984). Analyses of Survival Data. 24 , Chapman and Hall, New York.

5. Finkelstein M.S. A restoration process with dependent cycles, Automat. Remote Control , 53, 1115-1120, 1992. 
6. Finkelstein, M.S. (1999). Wearing-out components in variable environment, Reliability Engineering and System Safety, 66, N3, 235-242.

7. Finkelstein, M.S. (2000). Modeling a process of non-ideal repair. In: Recent advances in Reliability Theory. Limnios N., Nikulin M. (eds). Birkhauser, 4153.

8. Keiding, N. (1990). Statistical inference in the Lexis diagram. Philosophical Transactions of the Royal Society of London, A 332, 487-509.

9. Kijima M. (1989) Some results for repairable systems with general repair, $J$. Appl. Prob. , 26, 89-102.

10. Mair, W., Goymer, P., Pletcher, S., and Patridge, L. (2003). Demography of dietary restriction and death in Drosophila. Science, 301, 1731-1733.

11. Nelson, W. Accelerated Testing. John Wiley and Sons, New York, 1990.

12. Vaupel, J.W., and Yashin, A.I. (1987). Repeated resuscitation: how life saving alters life tables. Demography, 4, 123-135.

13. Vaupel, J.W., Carey, J.R., and Christensen, K. (2003). It's never too late. Science, 301, 1679-1681. 